\title{
DEAN RICKLES
}

\section{TIME, OBSERVABLES, AND STRUCTURE}

\begin{abstract}
In this chapter I consider what recent work on background independent physics can do for structuralism, and what structuralism can do for background independent physics. I focus on the problems of time and observables in gravitational physics. The 'frozen' character of the observables of general relativity is usually considered to constitute a serious problem for the theory. I argue that by invoking correlations between physical quantities we can provide a natural explanation of the appearance of time and change in timeless structures. I argue that this response can resolve a problem with Max Tegmark's 'extreme structuralist' position. I then consider what bearing the mathematical representation used (namely Rovelli's framework of 'partial' and 'complete' observables) has on the debate over the nature of structure in discussions of structural realism (i.e. the question of how structures are to be conceived). I argue that it has both the resources to ground the notion of structure in physics and to answer the 'no relations without relata' objection. ${ }^{1}$
\end{abstract}

1

\section{MATHEMATICAL STRUCTURE AND REALITY}

Mathematics, broadly speaking, is the science of patterns. Physics, broadly speaking, is the search for patterns in the natural world. Eugene Wigner's [16] famous conundrum concerning the "unreasonable effectiveness of mathematics in the natural sciences" constitutes an expression of puzzlement over the empirical success of physics, based as it is on mathematics. ${ }^{2}$ Put this way, of course, the problem has a rather simple answer: mathematics (the science of patterns) is so effective because the natural world (the subject matter of physics) is itself patterned. The regularities of physics are but instances of mathematical structures. For example, we can apply geometry to physical space because physical space has a structure that is (more or less) isomorphic to some geometrical structure (or a sequence of such structures, in the case of evolving 3-geometries, or geometrodynamics). We might, in somewhat different terms (and ignoring complications to do with representation), view our world as a model of the axioms of some systems of geometry (and of the axioms of quantum field theory, say-though this latter connection is rather more debatable).

However, the problem is really an old one, and there are some old solutions too. Pythagoras claimed, similarly, that there was no real distinction between 'the world of physics' and 'the world of mathematics'. Plato argued there was a very great difference: it amounted to concrete versus abstract, a distinction denied by Pythagoras. For Plato, of course, the concrete, physical world instantiated

\footnotetext{
${ }^{1}$ This chapter was originally written for the FQXi's Nature of Time essay competition (http:// www.fqxi.org/community/essay/winners/2008.1\#Rickles). I thank FQXi for permission to reproduce the essay (albeit in modified form) here.

${ }^{2}$ As he puts it: "the mathematical formulation of the physicist's often crude experience leads in an uncanny number of cases to an amazingly accurate description of a large class of phenomena" ([16], p. 230).
} 
(or 'partook of') the abstract forms (albeit imperfectly). This Platonic account is somewhat similar to the model-based view presented above.

More recently structural realists have answered the question about the effectiveness of mathematics by arguing that the empirical sciences are about the discovery of structural aspects of the world, and these structural aspects are of a mathematical nature (or, at least, of a kind that submit to mathematical representations). ${ }^{3}$ Radical 'ontic' structural realists turn this in to an all out ontological claim: science is about structure; scientific theories encode structural information about the world; and structure is all there is. In this way, structural realists aim to provide a complete story about how and why (mathematized) scientific theories work so well.

\section{EXTREME STRUCTURALISM}

Max Tegmark [14] has recently extended this basic structural realist idea by combining it with something like David Lewis' extreme brand of modal realism [6]. Not only is there the structure we observe (which is mathematical), there exist mathematical structures of all possible types! We have here, then, an extreme case of the principle of plenitude. Why would one ever wish to go to this extreme? To explain the nature and existence of the structure we observe. The laws (structure) of our universe are by no means necessary and so demand some explanation for why they are thus rather than so. Tegmark answers this question with absolute proliferation: our world is a mathematical structure in a multiverse of all possible structures. Interestingly, in one sense, though the structure we inhabit (and are, ourselves, part of) is itself contingent ${ }^{4}$, the existence of the structure we inhabit is in another sense necessary, since (being an instance of an eternal mathematical structure) it will be a possible world relative to all other worlds.

The maximal multiplicity of possible worlds, then, is utilized to ground a theory of everything that does not face problems of creation ex nihilo: mathematical structures are timeless, they are not the kinds of thing that can be created and destroyed. One can then, if one is so inclined, invoke an anthropic explanation of why we find ourselves in this particular mathematical structure. This is, admittedly, a hard view to swallow! However, if one wishes to explain why there is something rather than nothing (surely the ultimate explanandum?), then I see no

\footnotetext{
${ }^{3}$ Though not all structural realists would go this far. Some, for example, would prefer to say that structure is physical, and that there might be biological and social structures that are not necessarily mathematical. However, the recent trends are towards extending structural realism across the entire domain of science, including biology [5] and economics [12]. The distinction between 'physical' and 'mathematical,' if it can be established at all, is not quite as simple as it might seem prima facie-see Rickles [8] for a discussion of this difficulty in the context of string theory.

${ }^{4}$ For example, one can conceive of the laws being different, and indeed, as in David Lewis' theory, the existence of a plurality of structures of the sort described can provide the machinery to ground such possibilities.
} 
other alternative than to propose such curious-sounding theories as the invoking of eternally existing structures. If we are willing to accept this, then we are led to a belief in many types of (consistent) structure. If we are then further willing to view our world as one of these structures (i.e. literally a mathematical structure), then combined with the necessary anthropics, we have an explanation of what is often considered to be an insurmountable problem.

The major problematic step (by no means the only one), I take it, is that one requiring that we believe our world (ourselves included) is a mathematical structure. All aspects of reality in our world would have to be reconceptualized in terms of such structure. ${ }^{5}$ The common reaction to such a view bears similarities to Dr Johnson's attempted refutation of Berkeley's idealism by the kicking of a rock. In this case, the objection is that the world does not seem to be anything like a mathematical structure: mathematical structures are abstract and physical reality is concrete (whereupon you are invited to notice that there are spatiotemporally located, impermanent solid objects on which one could stub one's toe). But we have no way of knowing what it is like to be a mathematical structure: it could, after all, be just like this! Moreover, given our present knowledge of spacetime (on which more later), the idea that spatiotemporal location is such that it can serve to play so crucial a role as demarcating abstract from concrete seems absurd: the chronometrical structure of spacetime (including locations, conceived of as complexes of events) are themselves dynamically determined by laws of general relativity.

However, given this dynamical determination of spacetime structure, there are real problems in attempting to account for certain observed aspects of the world; time and change being cases in point. The world certainly appears to undergo change, and this, we usually (i.e. with our philosophy hats off) assume, must happen in time. How can time and change be part of a mathematical structure given that such structures are immutable and eternal? In the case of general relativity the structures appear to directly represent a world without time and change since the symmetries of the theory imply a zero Hamiltonian which, in the quantized theory, leads to a non-evolving Schrödinger equation.

In the remainder of this chapter I argue that recent work on problems of time and change in classical and quantum gravity can be brought to bear on the matter resulting in a satisfactory resolution. To deploy a Wheelerism, they show how one can have time without time. We can give an account (or, at least the outline of an account) of our world, qua mathematical structure, that at a fundamental level does not contain time. This account makes use of structuralism in a direct way. Thus, the account I present can be usefully incorporated into Tegmark's theory-or what

\footnotetext{
${ }^{5}$ Related to this is the problem of equivalent mathematical structures that correspond to distinct physical situations. In other words, one and the same structure can be taken to represent very different systems. I don't see this to be as problematic as it is sometimes taken to be. If there are indeed differences in the physical systems, then though we can indeed, in many cases, represent them using the same mathematical structure (for example, the Navier-Stokes equations can be applied to all manner of prima facie very different systems), that does not thereby mean that the systems would not have some other structures more closely corresponding to them. Any physical difference would simply mean that there ought to be a structural difference too, so long as we use a fine enough resolution of the structure.
} 
I shall call "ultrastructuralism"- -in order to defuse a major potential problem. As a bonus, as I mentioned above, one can extract a more physically motivated account of what structure is (at least in the context of physics).

\section{TIME AND SYMMETRY}

The universe, as a single object, is usually modelled as a four dimensional structure (a Lorentzian 4-geometry). ${ }^{6}$ This structure is naturally changeless: change happens within the universe, from one hypersurface (3-geometry) to another (with the time variable chosen arbitrarily on account of general covariance). At least, within our world it seems to be constructed in this way; the laws of general relativity themselves do not completely constrain the topology of space, and do not even constrain the dimensionality of the manifold.

Of course, general relativity leads us to view spacetime geometry as part of a coupled dynamical system, as something that satisfies equations of motion and coevolves with matter and radiation. But clearly the evolution here cannot be understood in an ordinary temporal sense, unless we have at our disposal some external time parameter against which to understand it. An alternative is to attempt to concoct some 'internal' parameter from the dynamical degrees of freedom that can then parametrize the evolution. The former 'external' parameter simply doesn't make sense in the context of general relativity. The internal parameter approach does not make sense at a global level, but local times can be established using appropriate invariants. However, even here, the notion of systems evolving against coordinate time is inappropriate: the time that emerges is a dynamical construction from events.

This, in a nutshell, is the problem of time in general relativity: spacetime geometry is a dynamical variable, but clearly the dynamics cannot be understood in the usual sense (that is, as involving an external time parameter). The problem is worse than this, however, and can trickle down from global (involving the universe as a whole and a timelessness that is fairly innocuous) to local (involving timelessness and changelessness of the states and observables within the universe). At the root of this problem is the symmetry group of general relativity, the group of four-dimensional diffemorphisms of the spacetime manifold. Diffeomorphism invariance makes local observables (i.e. observables sitting at spacetime points or within regions of spactime) an impossibility, for the equations of motion (of generally relativistic theories) are invariant with respect to diffeomorphisms that shift the points and regions about. Since there clearly are (in some sense) localized degrees of freedom, and these are what we observe (and that seem to evolve), we need some notion of local observable that does not make reference to the spacetime manifold but that fits our experience. That is, we need a background independent

\footnotetext{
${ }^{6}$ To this we might add various geometric object fields representing the observed matter and radiation.
} 
notion of observable that does not utilize external spatial and temporal parameters, for changes with respect to these will be symmetries of the theory.

A popular response is to use physical degrees of freedom to define observables and evolution. This can be understood as one kind of implementation of the 'internalist' strategy mentioned above. The observables so 'localized' are relational in the sense that they are not defined on a background space but only relative to other dynamical entities (matter fields, spatial volume, etc.). Observables are not of the form $\mathscr{A}(x, t)$ (where $x$ and $t$ label an independent manifold) but $\mathscr{A}(\mathscr{B})$ (where $\mathscr{B}$ is another observable and neither $\mathscr{B}$ nor $\mathscr{A}$ is privileged in any sense). One can then consider the relative evolution of such observables, looking at the way in which changes in the value of one are correlated with changes in the value of the other. This approach can give us notions of time and change that emerge as a consequence of functional relations between elements of a mathematical structure. However, this is to oversimplify matters: in order to properly appreciate the nature of this problem, and the suggested resolution ${ }^{7}$ I need to quickly cover the entangled concepts of gauge and constraints. I can then introduce Rovelli's framework for constructing suitably invariant observables, for constrained (gauge) systems, and show how it provides a structuralist response to the problem of time that can be utilized by the ultrastructuralist to explain time in an atemporal mathematical structure. $^{8}$

4

\section{CONSTRAINTS AND GAUGE}

The problems of time and change sketched above are aspects of the fact that general relativity is a gauge theory-its Hamiltonian formulation is given by constraints. The "gauge" here simply refers to the freedom in choosing coordinates used to parametrize space so that choosing a particular coordinate system amounts to choosing a gauge. Physical quantities are those that are independent of such a gauge choice. We give a very rough and ready presentation of these ideas herefor more details (in the context of the problem of time), see [11].

The diffeomorphism symmetry mentioned above affects the dynamics so that a standard Hamiltonian or Lagrangian formulation of the theory is not possible. Respectively, the canonical variables, $q$ and $p$, are not all independent (being required to satisfy identities known as constraints: $\phi(q, p)=0)$ and the Euler-Lagrange equations are not all independent. These identities serve to 'constrain' the set of phase space points that represent genuine physical possibilities: only those points

\footnotetext{
${ }^{7}$ This view has been defended by a variety of authors; most notably Bryce DeWitt and Carlo Rovelli. Here I adopt Rovelli's 'partial' and 'complete' observables formalism [13]. See [11] for a general review of the problem of time and proposed solutions.

${ }^{8}$ I restrict the discussion to classical systems in order to make the presentation easier to follow. For the technically savvy, one can transform to the quantum case, roughly, by thinking of the functional relation or correlation $\mathscr{A}(\mathscr{B})$ as representing the expectation values of $\mathscr{A}$ relative to the eigenvalues of $\mathscr{B}$.
} 
satisfying the constraints do so, and these form a subset in the full phase space known as the 'constraint surface'. This has a direct impact on the form of the observables. Since a pair of dynamical variables (not observables) that differ by a gauge transformation are indistinguishable, corresponding to one and the same physical state of affairs (the defining characteristic of a gauge transformation), the observables ought to register this fact too: that is, the observables of a gauge theory should be insensitive to differences amounting to a gauge transformation-as should the states in any quantization of such a theory: i.e. if $x$ is related to $y$ by a change of gauge, then states $\Psi$ must satisfy the gauge condition that $\Psi(x) \approx \Psi(y)$,

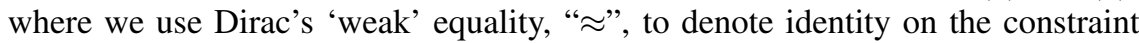
surface. ${ }^{9}$

More explicitly, where ' $\mathscr{A}$ ' is a dynamical variable, ' $O$ ' is the set of (genuine) observables, $x, y$ are states (represented by points on the constraint surface), and ' $\sim$ ' denotes gauge equivalence, we can express this as:

$$
\mathscr{A} \in \mathrm{O} \Longleftrightarrow(x \sim y) \supset(\mathscr{A}(x) \approx \mathscr{A}(y))
$$

Or, equivalently, we can say that the genuine observables are those dynamical variables that are constant on gauge orbits ' $[x]$ ' (where $[x]=\{y: y \sim x\}$ ):

(2) $\forall[x], \mathscr{A} \in \mathrm{O} \Longleftrightarrow \mathscr{A}[x]=$ const.

Most of the work done on constructing the observables of general relativity is done using the $3+1$ projection of the spacetime Einstein equations. That is, the constraints are understood as conditions laid down on the initial data $\langle\Sigma, h, K\rangle$ when we project the spacetime solution onto a spacelike hypersurface $\Sigma$-here, $h$ is a Riemannian metric on $\Sigma$ and $K$ is the extrinsic curvature on $\Sigma$; note that this formulation has since been superseded by a representation in terms of Wilson loops and their conjugate momenta (namely, fluxes). I won't go into the nitty gritty details here, but it turns out that the Hamiltonian of general relativity is a sum of constraints on this initial data (of the kind that generate gauge motions, namely 1st class)-hence, the dynamics is entirely generated by constraints and is therefore pure gauge. There is no evolution in time. This is the technical expression of the problems posed above and makes perfect sense if one thinks of time evolution as a diffeomorphism generating the shifting of data from one slice to another.

This formulation allows us to connect up the characterization of the observables to the dynamics (generated by constraints, abbreviated to $\mathscr{H}_{i}$ ) more explicitly:

$$
\mathscr{A} \in \mathrm{O} \Longleftrightarrow\left\{\mathrm{O}, \mathscr{H}_{i}\right\} \approx 0 \forall i
$$

In other words, the observables of the theory are those functions that have weakly vanishing (i.e. on the constraint surface) Poisson brackets with all of the (first-

\footnotetext{
${ }^{9}$ It seems that Einstein might have been aware of this implication soon after completing his theory of general relativity, for he writes that "the connection between quantities in equations and measurable quantities is far more indirect than in the customary theories of old" ([4], p. 71).
} 
class) constraints. ${ }^{10}$ These are the physical, gauge-invariant quantities: evolving with the constraints (the dynamics) does not generate a physically distinct state but simply changes the gauge.

A pressing problem in general relativity-especially pressing for constructing a quantum theory of gravity - is to find suitable entities that satisfy this formal definition. There are at least two types that fit the bill, both non-local in some way: (1) global quantities defined over the whole spacetime ${ }^{11}$ and (2) 'relational' quantities built out of correlations between field values and/or invariants. There seems to be some consensus forming that the latter type are the way to go, and these will serve as the appropriate vehicle for defining time in an unchanging mathematical structure, as well as defining the structures themselves.

5

\section{COMPLETE OBSERVABLES AS STRUCTURAL CORRELATIONS}

John Earman calls quantities of the form $\mathscr{A}(\mathscr{B})$ "coincidence occurrences". ${ }^{12}$ As he explains, "a coincidence occurrence consists in the corealization of values of pairs of (non-gauge invariant) dynamical quantities" ([2], p. 16). Earman thinks that this new conception of physical quantities signals the necessity of a shift from the traditional 'subject-predicate'-based ontologies, such as substantivalism and relationalism. I think this is the right thing to say, and have argued for and developed this point elsewhere (see $[9 ; 10 ; 11]$ ). It bears a striking resemblance to the version of structuralism espoused by Eddington:

any conception of structure (as opposed to substance) must be analysable into a complex of relations and relata, the relata having no structural significance except as the meeting point of several relations, and the relations having no significance except as connecting and ordering the relata. ([3], p. 121)

However, I spell it out rather differently. Rovelli's framework of partial and complete observables_-developed in [13] — provides, I think, the perfect formal framework in which to ground the conceptual framework.

- A partial observable is a physical quantity to which we can associate a measurement leading to a number

- A complete observable is defined as a quantity whose value (or probability distribution) can be predicted by the relevant theory.

\footnotetext{
${ }^{10}$ There are two types of constraint in general relativity: the Hamiltonian (or scalar) constraint and the momentum (or vector) constraint. These can be understood as encoding indeterminacy about 'when and where' some quantity is measured.

${ }^{11}$ There is a proof (for the case of closed vacuum solutions of general relativity) that there can be no local observables at all [15], where 'local' here means that the observable is constructed as a spatial integral of local functions of the initial data and their derivatives.

${ }^{12}$ We might also call them "Kretschmann observables" since they stem from Kretschmann's objection to general covariance later incorporated into Einstein's own 'point-coincidence' argument.
} 
Partial observables are taken to coordinatize an extended configuration space $\mathscr{Q}$ and complete observables coordinatize an associated (physical) reduced phase space $\Gamma_{\text {red }}$. The "predictive content" of some dynamical theory is then given by the kernel of the map $f: \mathscr{Q} \times \Gamma_{\text {red }} \rightarrow \mathbb{R}^{n}$. This space gives the kinematics of a theory and the dynamics is given by the constraints, $\phi\left(q^{a}, p_{a}\right)=0$, on the associated extended phase space $T^{*} \mathscr{Q}$.

The content appears to be this: there are quantities that can be measured whose values are not predicted by the theory. Yet the theory is deterministic (modulo quantum theoretic probabilities) because it does predict correlations between partial observables (i.e. complete observables). The dynamics is then spelt out in terms of relations between partial observables. Hence, the theory formulated in this way describes relative evolution of (non-gauge invariant) variables as functions of each other. No variable is privileged as the independent one (cf. [7], p. 5). The dynamics concerns the relations between elements of the space of partial observables, and though the individual elements do not have a well defined evolution, relations between them (i.e. correlations) do, and in such a way as to remain independent of coordinate space and time.

The interpretation vis-á-vis time is as follows: let $\phi=T$ be a partial observable parametrizing the ticks of a clock (laid out across a gauge orbit), and let $f=a$ be another partial observable (also spanning a gauge orbit). Both are non-gauge invariant quantities. A gauge invariant quantity, a complete observable, can (here borrowing from [1]) be constructed from these partial observables as:

$$
\mathscr{A}_{[f ; T]}(\tau, x)=f\left(x^{\prime}\right)
$$

These quantities encode correlations. They tell us what the value of a non-gauge invariant function $f$ is when, under the flow with respect to the vector field generated by the constraint, the non-gauge invariant function $T$ takes on the value $\tau$. This correlation is gauge invariant. These are the kinds of quantity that a background independent gauge theory like general relativity is all about. We don't talk about the value of the gravitational field at a point of the manifold, but where some other physical quantity (say, a value of the electromagnetic field) takes on a certain value. In this sense, this formal framework codifies Eddington's earlier views, and also Earman's characterisation of coincidence occurrences. ${ }^{13}$ I prefer to think of the complete observables as primitive structural correlations that are reducible only in unphysical ways (corresponding to distinct gauge choices).

\section{6}

\footnotetext{
${ }^{13}$ Once again, we find that Einstein was surprisingly modern-sounding on this point, writing that "the gravitational field at a certain location represents nothing 'physically real,' but the gravitational field together with other data does" ([4], p. 71). Likewise, the "other data" will represent nothing without yet more data (such as the gravitational field). The correlations are the fundamental things.
} 


\section{RELATIONS WITHOUT RELATA}

Let us return to the issue of structuralism. Epistemic structural realists argue that the best we can hope for is to get to know structural aspects of the world, since we only ever get to observe relational rather than intrinsic properties (in our experiments and so on). However, in a background independent gauge theory like general relativity we have seen that the physical observables just are relational quantities: this is all there is! One cannot even speak of an independent spacetime. Hence, the notion of a non-relational quantity, defined at a point of spacetime, is physically incoherent, though we can provide a purely formal expression of the notion in terms of gauge variant coordinate-dependent quantities.

In other words, there's nothing 'underneath' the relational properties (as encoded in the 'overlapping' dynamical fields), so that these exhaust what there is, leading to an ontological structuralism motivated entirely by the proper conceptualisation (and formalisation) of general relativity. Hence, we have here an empirical argument for ontic structural realism that evades the standard 'no relations without relata' objection. The relations are the correlations here (the gauge invariant, complete observables), and the 'relata' would be the non-gauge invariant, partial observables. But the partial observables being non-gauge invariant do not correspond to physical reality: only the complete observables do. Partial observables correspond to an arbitrary choice of gauge that can be transformed away. We cannot decompose the correlations in an ontological sense, though we clearly can in a epistemic or formal sense-indeed, the correlates constitute our 'access points' to the more fundamental, physical correlations. We talk about correlations in terms of quantities that are correlated. But there is a clear ontological division between the status of partial observables (i.e. relata) and complete observables (i.e. relations). This is, then, precisely why we face problems regarding the 'subjectpredicate'-style ontologies that Earman mentions: there are no independent subjects that are the 'bearers' of properties and the 'enterers' of relations. We can seemingly invoke such subjects in our representations, but it is mere artifice.

What is interesting about this version of ontic structural realism is its independence from standard philosophy of science issues. We do not need to ground the position in the underdetermination of theory by data or the problem of ontological revisions at the level of objects. The method of motivation is entirely internal to the interpretation of mature physical theory.

\section{CONCLUSION}

The position I have described involves the idea that physical systems (which I take to be characterized by the values for their observables) are exhausted by extrinsic or relational properties: they have no intrinsic, local properties at all! This is a curious consequence of background independence coupled with gauge invariance and 
leads to a rather odd picture in which objects and structure are deeply entangled. Inasmuch as there are objects at all, any properties they possess are structurally conferred: they have no reality outside some correlation. What this means is that the objects don't ground the structure; they are nothing independently of the structure, which takes the form of a (gauge-invariant) correlation between (non-gauge invariant) field values. With this view one can both evade the standard 'no relations without relata' objection and the problem of accounting for the appearance of time (in a timeless structure) in the same way.

\section{BIBLIOGRAPHY}

[1] Dittrich, B. (2007). Partial and Complete Observables for Hamiltonian Constrained Systems. General Relativity and Gravitation 39: 1891-1927.

[2] Earman, J. (2006). The Implications of General Covariance for the Ontology and Ideology of Spacetime. In D. Dieks (ed.), The Ontology of Spacetime (pp. 3-23). Elsevier.

[3] (1921) A Generalisation of Weyl's Theory of the Electromagnetic and Gravitational Fields. Proceedings of the Royal Society of London. Series A 99(697): 104-122.

[4] Einstein, E. (1918). Dialogue about objections to the theory of relativity. In A. Engel, editor, The Collected Papers of Albert Einstein, Vol. 7, The Berlin Years: Writings: 1918-1921, English Translation of Selected Texts. Princeton University Press, 2002.

[5] French, S. (2011) Shifting to Structures in Physics and Biology: A Prophylactic for Promiscuous Realism. Studies in the History and Philosophy of Biology and the Biomedical Sciences 42(2): 164-73.

[6] Lewis, D. K. (1986). On the Plurality of Worlds. Blackwell.

[7] Montesinos, M., C. Rovelli, and T. Thiemann. (1999). An SL(2, R) Model of Constrained Systems with two Hamiltonian Constraints. Physical Review D, 60: 044009.

[8] Rickles, D. (2010) Mirror Symmetry and Other Miracles in Superstring Theory. Foundations of Physics. Online First: DOI: 10.1007/s10701-010-9504-5.

[9] Rickles, D. (2008). Who's Afraid of Background Independence. In D. Dieks (ed.), The Ontology of Spacetime II (pp. 133-152). Elsevier.

[10] Rickles, D. (2007). Symmetry, Structure, and Spacetime. North Holland: Elsevier.

[11] Rickles, D. (2006). Time and Structure in Canonical Gravity. In D. Rickles, S. French, and J. Saatsi (eds.), The Structural Foundations of Quantum Gravity (pp. 152-195). Oxford University Press.

[12] Ross, D. (2008). 'Ontic Structural Realism and Economics. Philosophy of Science 75: 731-741.

[13] Rovelli, C. (2002. Partial Observables. Physical Review D 65: 124013-124013-8.

[14] Tegmark, M. (2006). The Mathematical Universe. Foundations of Physics 38: 101-150.

[15] Torre, C. G. (1993). Gravitational Observables and Local Symmetries. Physical Review D 48: R2373-R2376.

[16] Wigner, E. P. (1967). The Unreasonable Effectiveness of Mathematics in the Natural Sciences. In E. P. Wigner (ed.), Symmetries and Reflections: Scientific Essays (pp. 222-237). Indiana University Press. 